Int. J. Dev. Biol. 57: 35-39 (2013)

doi: $10.1387 / \mathrm{ijdb} .120125 \mathrm{mz}$

\title{
The NOBOX protein becomes undetectable in developmentally competent antral and ovulated oocytes
}

\author{
MARTINA BELLI 1 ,\#, DANILO CIMADOMO ${ }^{1, \#, ~ V A L E R I A ~ M E R I C O ~}{ }^{1}$, CARLO A. REDI ${ }^{1}$, \\ SILVIA GARAGNA ${ }^{1,2,3, *}$ and MAURIZIO ZUCCOTTII,*
}

\begin{abstract}
'Laboratorio di Biologia dello Sviluppo, Dipartimento di Biologia e Biotecnologie 'Lazzaro Spallanzani', Universita 'degli Studi di Pavia, Pavia, ${ }^{2}$ Centro di Ingegneria Tissutale, Universita' degli Studi di Pavia, Pavia, ${ }^{3}$ Centro di Eccellenza in Biologia Applicata, Universita' degli Studi di Pavia, Pavia and 'Unità di Anatomia, Istologia ed Embriologia, Dipartimento di Scienze Biomediche, Biotecnologiche e Traslazionali (S.Bi.Bi.T.), Universita' degli Studi di Parma, Italy
\end{abstract}

\begin{abstract}
The oocyte-specific NOBOX protein is an important player during oocyte growth. Its absence in Nobox-/- mice arrests the transition from primordial to growing follicles and downregulates the expression of a number of genes, including Oct4, a transcription factor crucial in the acquisition of oocyte developmental competence. Despite its role during folliculogenesis, a clear description of the expression of NOBOX throughout oocyte growth is lacking. Here, we have analysed the pattern of expression of both the Nobox gene (qRT-PCR) and its protein (immunofluorescence) during folliculogenesis, classifying the oocytes based on their size (six classes: 10-30, 31-40, 41$50,51-60,61-70,71-80 \mu \mathrm{m}$ ) and chromatin organisation (NSN, Non Surrounded Nucleolus or SN, Surrounded Nucleolus). Significant differences were observed in Nobox transcription in the group of 41-50 $\mu \mathrm{m}$ (NSN > SN), 71-80 $\mu \mathrm{m}$ (NSN > SN) and in developmentally incompetent metaphase II-derived NSN (MIINSN) or competent metaphase II-derived SN (MIISN) oocytes (MIINSN $>$ MIISN). The NOBOX protein is expressed throughout oocyte growth in the nucleus of ovarian NSN and in MIINSN oocytes; in contrast, beginning with SN oocytes of 61-70 $\mu \mathrm{m}$, it becomes almost undetectable. Our data, while being in line with the hypothesis of a regulative role of NOBOX on Oct4 gene expression at the primordial/primary stage, when both transcription factors are coincidentally expressed, also indicate that this role might not be maintained in the subsequent growing stages. Furthermore, the sharp difference of NOBOX expression in developmentally incompetent or competent oocytes makes this protein a putative marker of their quality.
\end{abstract}

KEY WORDS: NOBOX, oocyte growth, oocyte developmental competence, folliculogenesis

\section{Introduction}

The oocyte-specific transcription factor NOBOX is an important player during oocyte maturation. Down-regulation of its expression in Nobox ${ }^{1-}$ mice blocks the transition from primordial to growing follicles and down-regulates the expression of a number of genes such as H10o, Gdf9, Bmp15, Dnmt1o, Zar1, Fgf8, Rfp14, Mos and Oct4 (Rajkovic et al., 2004). NOBOX has a high affinity, when tested using competition Electrophoretic Mobility Shift Assay, for a binding element present in the promoter region of the mouse Oct4, suggesting a direct role for NOBOX in the positive regulation of its transcription (Choi \& Rajkovic 2006).

The OCT4 protein is expressed in primordial/primary oocytes, then it is down-regulated and it reappears again at the time of follicle recruitment (Zuccotti et al., 2009a), but only in those oocytes whose nucleus presents a ring of heterochromatin surrounding the nucleolus (surrounded nucleolus, SN oocytes). Instead, the protein is down-regulated, almost undetectable, in oocytes that

Abbreviations used in this paper: MII, metaphase II; MIINSN, metaphase II oocytes derived from NSN antral oocytes; MIISN, metaphase II oocytes derived from SN antral oocytes; NSN, non-surrounded nucleolus; SN, surrounded nucleolus.

\footnotetext{
*Address correspondence to: Maurizio Zuccotti. Unità di Anatomia, Istologia ed Embriologia, Dipartimento di Scienze Biomediche, Biotecnologiche eTraslazionali Universita' degli Studi di Parma, Via Volturno 39, 43125, Parma, Italy. e-mail: maurizio.zuccotti@ unipr.it or Silvia Garagna. Laboratorio di Biologia dello Sviluppo, Dipartimento di Biologia e Biotecnologie 'Lazzaro Spallanzani', Universita` degli Studi di Pavia, Via Ferrata 9, 27100, Pavia, Italy. e-mail: silvia.garagna @ unipv.it
}

\#Note:These authors have contributed equally to this work.

Supplementary Material (3 figures) for this paper is available at: http://dx.doi.org/10.1387/ijdb.120125mz

Accepted: 7 January 2013. Final, author-corrected PDF published online: 8th March 2013.

ISSN: Online 1696-3547, Print 0214-6282

(c) 2013 UBC Press

Printed in Spain 
lack of this ring (non surrounded nucleolus, NSN oocytes). This profile of expression is maintained throughout oocyte growth, up to the fully-grown SN and NSN antral oocytes and in their in vitro derived MIINSN and MIISN oocytes (Zuccotti et al., 2008, 2009b). An interesting characteristic of the $\mathrm{MII}^{\mathrm{NSN}}$ and MII ${ }^{\mathrm{SN}}$ oocytes is that only the latter may reach full term development, whereas the former arrest development at the 2-cell stage (Zuccotti et al., 1998, 2002a; Inoue et al., 2008).

Despite the interest in the relationship between NOBOX and Oct4 during folliculogenesis, up to date a clear description of the profile of expression of the NOBOX protein throughout oocyte growth in developmentally incompetent NSN or competent SN oocytes has not yet been given. In the present study we analysed the profile of expression of both the Nobox gene (quantitative real-time PCR, qRT-PCR) and its protein (immunofluorescence) in NSN and SN oocytes isolated from primary to fully-grown follicles and in their derived MIINSN and MIISN oocytes.

\section{Results}

\section{The transcription profile of the Nobox gene increases during oocyte growth}

Using qRT-PCR, we determined the profile of Nobox expression throughout oocyte growth, from 10-30 $\mu \mathrm{m}$ in diameter (set at 1) to fully-grown NSN or SN and in their derived MII ${ }^{\text {SSN }}$ or MIISN oocytes. Fig. 1 illustrates an increasing number of Nobox transcripts in NSN oocytes during the transition from 10 to $40 \mu \mathrm{m}$ in diameter $(p=0.03)$ and, during growth, in both NSN and SN oocytes, reaching a 7-fold peak in oocytes of 61-70 $\mu \mathrm{m}$ (NSN, p <0.001; SN, $\mathrm{p}=0.005)$. Then, in fully-grown NSN and SN oocytes $(71-80 \mu \mathrm{m}$ in diameter), Nobox transcripts decrease significantly $(p<0.001)$ down to 5- or 4-fold, respectively and remain constant in their derived MII oocytes. When comparing NSN vs. SN oocytes within each single size class, significant differences were observed in the group of 41-50 $\mu \mathrm{m}$ (NSN > SN, p=0.003), 71-80 $\mu \mathrm{m}$ (NSN > $\mathrm{SN}, \mathrm{p}=0.017$ ) and MII oocytes (MIINSN $\left.>\mathrm{MII}^{\mathrm{SN}}, \mathrm{p}=0.004\right)$. Nobox expression in ovulated MII oocytes was similar to that of developmentally competent $\mathrm{MII}{ }^{\mathrm{SN}}(\mathrm{p}=0.67)$ and significantly lower than that of developmentally incompetent MIINSN oocytes $(p=0.002)$.

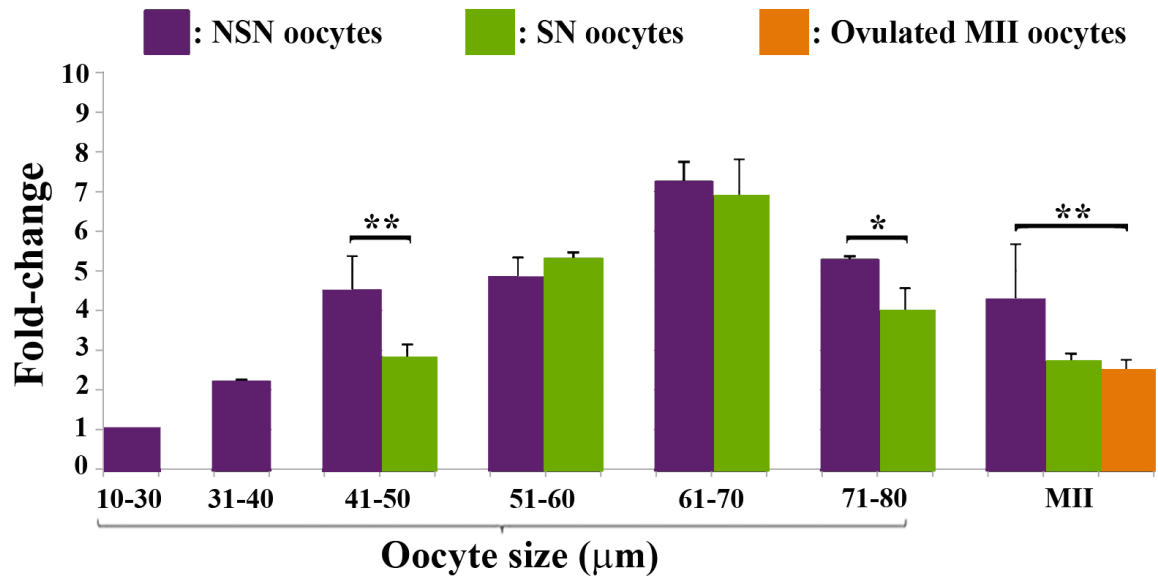

Fig. 1. Fold-change representation of the number of Nobox transcripts during oocyte growth and in MII oocytes. Expression of the gene in oocytes of 10-30 $\mu \mathrm{m}$ in diameter is set at $1 .{ }^{*}, p<0.05 ;{ }^{* *}, p<0.01$.
Interestingly, the highest expression variability in all the classes analysed was detected in MIINSN oocytes, as observed also for a number of other gene transcripts (Gentile et al., 2004). This might be explained with the presence of intermediate forms of NSN oocytes that show a trend towards the aggregation of the Hoechst-positive heterochromatin around the nucleolus, although lacking of a ring (Longo et al., 2003).

\section{NOBOX protein is present throughout folliculogenesis and is down-regulated in antral SN, MIISN and ovulated MII oocytes}

Fig. $2 \mathrm{~A}-\mathrm{C}$ shows the presence of the NOBOX protein confined within the nucleus of oocytes with a size in diameter comprised between 10 to $40 \mu \mathrm{m}$, in line with the role played by NOBOX during the transition from the primordial to the primary stage of maturation (Rajkovic et al., 2004). At the time of follicle recruitment (41-50 $\mu \mathrm{m})$, when the SN-type of chromatin organisation first appears, both NSN and SN oocytes present NOBOX within their nucleus. Although the most representative pattern of expression is that reported in Fig. 2 (D-F: NSN, 74\%; S-U: SN, 70\%), we also observed the presence of oocytes with different patterns (Fig. 1S). In the following class (51-60 $\mu \mathrm{m}$; Fig. 2 G-I; V-X), both NSN and SN oocytes showed a positive fluorescence signal in the nucleus.

Then, a major change occurred in oocytes of 61-70 $\mu \mathrm{m}$ in diameter. Whilst NSN oocytes maintained their nuclear NOBOX localisation, in $\mathrm{SN}$ oocytes the protein was markedly downregulated, almost undetectable, with the exception of one/three small perinucleolar areas (arrow, Fig. 2 Z,A'). This decreased fluorescence was even more evident in the following class of fullygrown oocytes (71-80 $\mu \mathrm{m})$. Again, in SN oocytes, the presence of NOBOX was limited to one/three small perinucleolar domains (arrows, Fig. 2 C',D') characterised by the absence of Hoechstpositive chromatin, as shown by $3 \mathrm{D}$ analysis (Fig. 2S). In this class that precedes ovulation, we could observe the presence of oocytes $(21 \%)$ with a chromatin conformation intermediate between the NSN and SN form (Longo et al., 2003), namely with a ring surrounding the nucleolus, but also with dispersed chromatin typical of an NSN oocyte (Fig. 3). Interestingly, the distribution of the NOBOX protein whilst progressively decreasing within the nucleus in the intermediate chromatin conformation (Fig. $3 \mathrm{E}, \mathrm{H}$ ), it was restricted to small foci around the nucleolus in SN oocytes (Fig. 3 F,I).

When matured in vitro to the MII stage, only MIINSN oocytes presented NOBOX within the whole cell volume (Fig. 2Q), suggesting a release of the protein in the ooplasm during germinal vesicle breakdown; instead, in MIISN (Fig. 2F') and in ovulated MII oocytes (Figure $3 \mathrm{~S}$ b) NOBOX was down-regulated.

\section{Discussion}

NOBOX is a transcription factor central to the transition from primordial to growing oocytes (Rajkovic et al., 2004). Whilst the expression of the Nobox gene has already been described in a number of earlier studies in both mouse (Suzumori et al., 2002; Rajkovic et al., 2004; Choi and Rajkovic, 2006; Choi et al., 2010) and human (Huntriss et al., 2006; Qin et al., 2007), 
Fig. 2. Expression of the NO$B O X$ protein (green) in the nucleus $\left(A, D, G, J, M, S, V, Y, B^{\prime}\right)$ of ovarian oocytes classified according to their size in diameter and depending on their NSN or SN chromatin configuration. In MIINSN Oocytes (P), NOBOX is detected in the cytoplasm; whereas in MIISN (E') it is undetectable. Arrowhead, NOBOX is localised as small foci around the nucleolus; nuclei were counterstaining with DAPI (blue); bar, $5 \mu \mathrm{m}$.

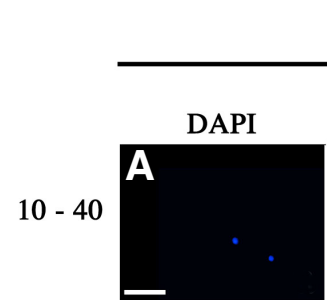

NSN
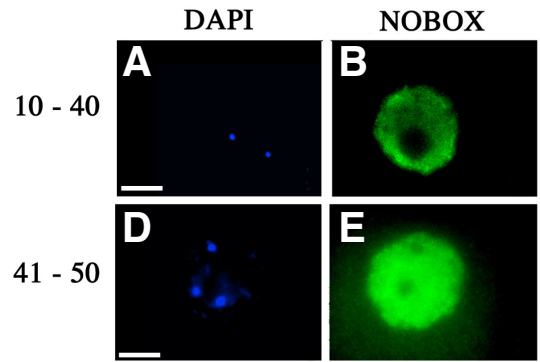

$51-60$

\section{$\mathbf{G}$}
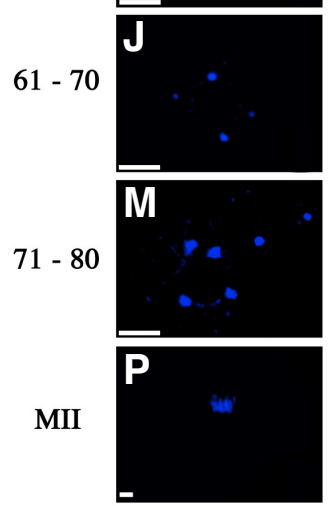
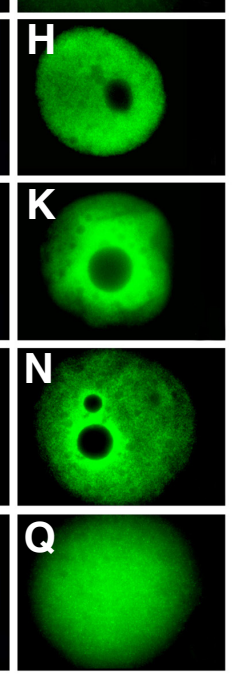
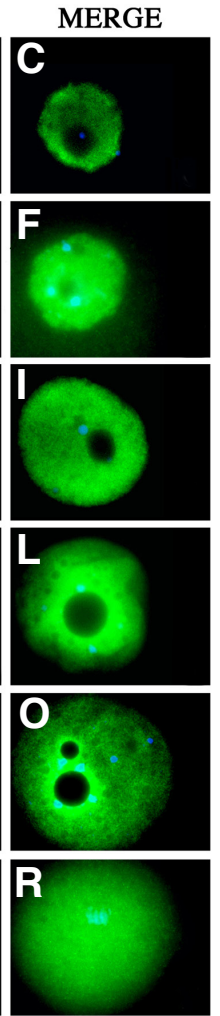

SN

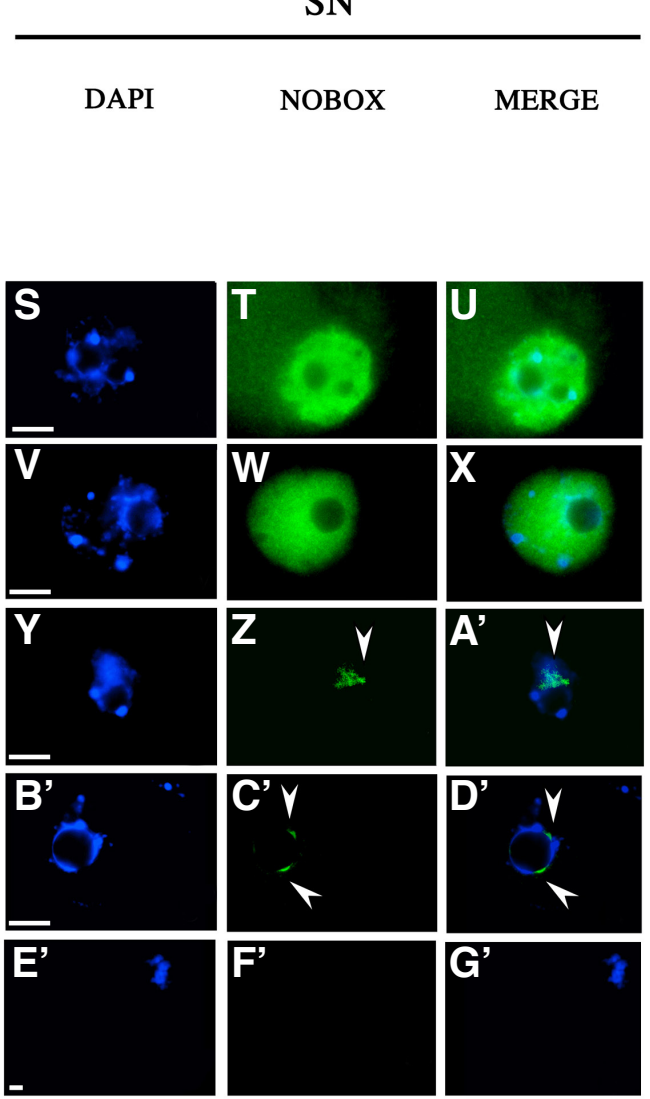

the presence of the NOBOX protein throughout oocyte growth in the adult ovary has never been shown before. The acquisition of this knowledge is central to the understanding of the regulatory role, attributed to the NOBOX protein, upstream of a number of genes crucial to oocyte growth e.g., Oct4, Gdf9, Bmp15, Fgf8, H1oo, Mos, Rfpl4, Zar1 and Dnmt1o (Rajkovic et al., 2004; Choi and Rajkovic, 2006). Thus, in the present study, we give the first account of the pattern of expression of the oocyte-specific NOBOX protein during folliculogenesis. Whilst its expression at the time of primordial to primary transition confirmed previous results (Rajkovic et al., 2004), the presence of this protein in oocytes beyond the primary stage is novel. Furthermore, this is the first report that correlates the presence of the NOBOX protein with the female gamete developmental competence or incompetence. In developmentally incompetent NSN oocytes the protein is detected throughout growth; on the contrary, at the latest stages of maturation of developmentally competent SN oocytes its expression is down-regulated.

Earlier studies have shown that Nobox knockdown down-regulates the expression of a number of key genes in oogenesis, including the transcription factor Oct4(Rajkovic et al., 2004). Whilst our data are in line with the hypothesis of a NOBOX positive transcriptional regulation of the Oct4 gene at the primordial/primary stage (Choi and Rajkovic, 2006), when both transcription factors are simultaneously present (Zuccotti et al., 2009a), they also indicate that this regulative role might not be maintained in the following stages of oocyte growth. In fact, while developmentally competent $\mathrm{SN}$ oocytes are characterised by the presence of OCT4 (Zuccotti et al., 2009a) and the lack of NOBOX, developmentally incompetent NSN oocytes show a reverse pattern of the two transcription factors.

The described sharp up- or down-regulation of NOBOX makes this protein, together with OCT4 and STELLA(Zuccotti et al., 2008, 2009a,b), a putative marker of the mouse
Fig. 3. Antral oocytes with an NSN-, intermediate- and SN-form of chromatin organisation. Within the class of fully-grown antral oocytes $(>70 \mu \mathrm{m})$, we observed an intermediate form of chromatin organisation (B) with a lower intensity pattern of NOBOX expression $(\mathbf{E}, \mathbf{H})$ when compared to NSN (D,G). Bar, $5 \mu \mathrm{m}$. 
oocyte quality that could now be tested in human and species of economic interest.

\section{Materials and Methods}

\section{Animals}

Four to six week-old B6C3F1 female mice were purchased from Charles River (Como, Italy) and maintained in the department animal facility under controlled conditions: $21^{\circ} \mathrm{C}$ of temperature and a dark/light cycle of 12/12 hours. Research on mice has been performed after the approval of the Animal Ethics Committee of the University of Pavia. Animals were maintained according to the Guide for Care and Use of Laboratory Animals.

\section{Hormonal treatment}

Females were injected intraperitoneally with 3.75 IU Folligon (Intervet Italia, Segrate, Italy) $48 \mathrm{hr}$ before sacrifice. For the isolation of ovulated MII oocytes, females were injected intraperitoneally with 3.75 IU Folligon, followed, $48 \mathrm{hr}$ later, by an injection with 3.75 IU Corulon (Intervet Italia, Segrate, Italy). Ovulated MIl oocytes were isolated from the oviducts 15 hr after Corulon injection.

\section{Oocyte isolation and classification}

Oocytes with a size in diameter comprised between 10-70 $\mu \mathrm{m}$ were isolated as follows: after isolation, ovaries were placed in a glass-dish containing $1 \mathrm{ml} \mathrm{M2} \mathrm{BSA-free} \mathrm{medium} \mathrm{supplemented} \mathrm{with} 1000 \mathrm{IU} / \mathrm{ml}$ collagenase type II (Sigma-Aldrich, Milano, Italy, cat. N. C6885), minced into small pieces, transferred in $1.5 \mathrm{ml}$ tubes and incubated for $5 \mathrm{~min}$ at $37^{\circ} \mathrm{C}$. Then, the tubes were vortexed for $2 \mathrm{~min}$ and incubated again for further $13 \mathrm{~min}$. Five-hundred microlitres of $1 \%$ bovine serum albumin (BSA; Sigma-Aldrich, Milano, Italy, cat. N. A3311) was added in order to block the enzymatic activity. The tubes were further vortexed ( $2 \mathrm{~min})$, centrifuged (2000 $\mathrm{g}$ for $2 \mathrm{~min}$ ), the pellet resuspended in $1.5 \mathrm{ml}$ M2 BSA-free medium containing $2.4 \mathrm{mM}$ EDTAand incubated for $3 \mathrm{~min}$ at $37^{\circ} \mathrm{C}$. To release oocytes from their follicles, the suspension was vortexed $(1 \mathrm{~min})$ and centrifuged (2000 $\mathrm{g}$ for $2 \mathrm{~min}$ ). The pellet was then resuspended in $1 \mathrm{ml} \mathrm{M} 2$ medium containing $0.4 \%$ BSA and transferred at the bottom of a glass-dish. Under a stereomicroscope (Olympus SZX9, Olympus Italia, Segrate, Italy) and using a hand-pulled Pasteur micropipette, oocytes with a size comprised between 10-70 $\mu \mathrm{m}$ were isolated and transferred in a $20 \mu \mathrm{l}$ drop of M2 medium (0.4\% BSA). Under an inverted microscope (Olympus CK40) and using a 10X ocular micrometer, oocytes were assigned to 5 classes depending on their size in diameter: class I, 10-30 $\mu \mathrm{m}$; class II, 31-40 $\mu \mathrm{m}$; class III, 41-50 $\mu \mathrm{m}$; class IV, 51-60 $\mu \mathrm{m}$ and class V, 61-70 $\mu \mathrm{m}$. Fully-grown antral follicles, containing oocytes with a size in diameter $>70 \mu \mathrm{m}$ (class VI) were punctured from the ovary using a sterile insulin needle in M2 medium $(0.4 \%$ BSA). Ooctyes were freed from follicle cells by pipetting in and out through a mouth-controlled hand-pulled Pasteur micropipette.

MII ovulated oocytes were isolated from the oviducts in M2 medium containing $500 \mathrm{IU} / \mathrm{ml}$ hyaluronidase type II (Sigma-Aldrich, Milano, Italy, cat. $\mathrm{N}$. H2126) to remove cumulus cells. Oocytes were washed in M2 medium $(0.4 \% \mathrm{BSA})$ and either transferred in lysis buffer for qRT-PCR analysis or fixed in $4 \%$ paraformaldehyde (PFA) in $1 \mathrm{X}$ PBS for immunofluorescence (see below).

\section{Oocyte classification based on chromatin organisation}

Following classification based on their size in diameter, oocytes were transferred into $20 \mu \mathrm{l}$ droplets of $\mathrm{M} 2$ medium $(0.4 \% \mathrm{BSA})$ containing Hoechst 33342 (Sigma-Aldrich, Milano, Italy, cat. N. B2261) at room temperature for 15 min and further grouped on the basis of their NSN or SN chromatin organisation (Zuccotti et al., 1995). The Hoechst concentration varied depending on the class: $0.15 \mu \mathrm{g} / \mathrm{ml}$ for class III; $0.08 \mu \mathrm{g} / \mathrm{ml} \mathrm{Hoechst} 33342$ for classes IV and V; $0.05 \mu \mathrm{g} / \mathrm{ml}$ for fully-grown antral oocytes (>70 $\mu \mathrm{m})$. Each single oocyte was then transferred into a $4 \mu \mathrm{l}$ droplet of $\mathrm{M} 2$ medium $(0.4 \%$ $\mathrm{BSA}$ ) and NSN/SN classified under a fluorescence microscope (Olympus AX70) at 10X magnification with UV fluorescence light (Zuccotti etal., 1995).

\section{In vitro maturation of fully-grown antral oocytes to the MII stage}

Following isolation and classification, NSN and SN fully-grown antral oocytes were rinsed and transferred into drops of $\alpha$-MEM medium (Life Technologies, Monza, Italy, cat. N. M4526) containing 5\% heat-inactivated fetal bovine serum (FBS; Life Technologies, Monza, Italy, cat. N. 10270106), 2 mM L-Glutamine (Life Technologies, Monza, Italy, cat. N. 25030), 5 mM Taurine (Sigma-Aldrich, Milano, Italy, cat. N. T0625) and $26 \mu \mathrm{g} / \mu \mathrm{l}$ sodium pyruvate (Sigma-Aldrich, Milano, Italy, cat. N. P4562). Oocytes were then incubated at $37^{\circ} \mathrm{C}$ for $15 \mathrm{hr}$ under a humidified atmosphere of $5 \% \mathrm{CO}_{2}$, $5 \% \mathrm{O}_{2}$ and $90 \% \mathrm{~N}_{2}$.

\section{RNA extraction, retrotranscription and qRT-PCR}

Following classification, single ovarian NSN, SN, MII ${ }^{\mathrm{NSN}}, \mathrm{MII}{ }^{\mathrm{SN}}$ or ovulated MIl oocytes were transferred, together with $1 \mu \mathrm{IM} 2$ medium $(0.4 \% \mathrm{BSA}$ ), into $0.2 \mathrm{ml} \mathrm{PCR}$ tubes containing $1.8 \mu \mathrm{l}$ lysis buffer (Zuccotti et al., 2002b) and stored at $-20^{\circ} \mathrm{C}$ for at least $12 \mathrm{hr}$. One picogram $(1 \mu \mathrm{l})$ of luciferase control RNA (Promega, Milano, Italy, cat. N. L4561) (used as an exogenous marker on which the results were normalised) was added to each tube (Thelie et al., 2009), together with $17 \mu$ l of retrotranscriptase mixture (Life Technologies, Monza, Italy): 1X 10X-Buffer, $5 \mathrm{mM} \mathrm{MgCl}$, $4 \mathrm{mM}$ each dNTPs, $0.625 \mu \mathrm{M}$ Oligo d(T $)_{16}, 1.875 \mu \mathrm{M}$ Random Hexamers, 20 IU RNase inhibitor, 50 IU MuLV reverse transcriptase. The amplification reaction was performed on the Rotor-Gene 6000 thermocycler (Corbett Research, Sidney, Australia) at $25^{\circ} \mathrm{C}$ for $10 \mathrm{~min}, 42^{\circ} \mathrm{C}$ for $60 \mathrm{~min}, 99^{\circ} \mathrm{C}$ for $5 \mathrm{~min}$. The presence of the oocyte within each tube has been confirmed analyzing the expression of the Hprtgene (forward primer: CCTGCTGGATTACATTAAAGCACT; reverse primer: GTCAAGGGCATATCCAACAACAAAC; amplicon size: $352 \mathrm{bp}$ ) on $3 \mu \mathrm{l}$ of cDNA, using the Applied Biosystems GeneAmp 9700 thermocycler (Life Technologies, Monza, Italy) under the following PCR conditions: $94^{\circ}$ $\mathrm{C}$ for $5 \mathrm{~min}, 40$ cycles of $94^{\circ} \mathrm{C}$ for $30 \mathrm{sec}, 53^{\circ} \mathrm{C}$ for $30 \mathrm{sec}, 72^{\circ} \mathrm{C}$ for 30 sec and a final step at $72^{\circ} \mathrm{C}$ for $7 \mathrm{~min}$ and cooling to $4^{\circ} \mathrm{C}$. PCR products were run on a $2 \%$ agarose gel and observed with the BioRad Gel Doc 2000. $97.5 \%$ of the samples resulted positive for the presence of the Hprt transcript (data not shown) and were processed further.

The cDNA derived from 10 single oocytes of the same size class and chromatin organisation were pooled together and analysed by qRT-PCR. Data of each class of oocytes were obtained from three independent experiments. Five microlitres of cDNA were added to a total of $15.0 \mu \mathrm{l}$ reaction mix (Diatech Pharmacogenetics srl, Jesi, Ancona, Italy) containing 1X Master mix and either $150 \mathrm{nM}$ of Nobox forward primer (AAAGACCCGAACCCTGTACC), $150 \mathrm{nM}$ Nobox reverse primer (GGAAATCTCATGGCGTTTGT) or $200 \mathrm{nM}$ Luciferase forward primer (AGTCGATGTACACGTTCGTCA), $200 \mathrm{nM}$ Luciferase reverse primer (CAGTGCAATTGTTTTGTCACG), brought to volume with MilliQ water (Merck-Millipore, Vimodrone, Milano, Italy). The amplification reaction was performed on a Rotorgene 6000 thermocycler with the following program: $95^{\circ} \mathrm{C}$ for $5 \mathrm{~min}, 38$ cycles at $95^{\circ}$ $\mathrm{C}$ for $10 \mathrm{sec}, 62.5^{\circ} \mathrm{C}$ for $20 \mathrm{sec}, 72^{\circ} \mathrm{C}$ for $20 \mathrm{sec}$. The qRT-PCR results of three independent experiments were processed with the SigmaStat 3.5 software using the one-way ANOVA test.

\section{Immunofluorescence analysis}

Oocytes of class IV $(51-60 \mu \mathrm{m})$, class V $(61-70 \mu \mathrm{m})$ and class VI (> 70 $\mu \mathrm{m})$ were fixed with $4 \%$ PFA in $1 \mathrm{X}$ PBS for $30 \mathrm{~min}$ at $4^{\circ} \mathrm{C}$. After fixation cells were washed in PBT [0.1\% Tween-20 (Sigma-Aldrich, Milano, Italy, cat. N. P1379) in 1 X PBS]. Permeabilization has been done in $0.5 \%$ Triton X-100 (Sigma-Aldrich, Milano, Italy, cat. N. X-100) in 1X PBS for 15 min, followed by incubation for $1 \mathrm{hr}$ in $0.1 \%$ BSA in PBT at $37^{\circ} \mathrm{C}$. Immunostaining was performed with a rabbit polyclonal anti-mouse-NOBOX polyclonal antibody in 1 X PBS at $4^{\circ}$ C overnight (1:500; Abcam, Cambridge, UK, cat. N. ab41521). After 3 washes in PBT of 15 min each, samples were incubated at $37^{\circ} \mathrm{C}$ for $45 \mathrm{~min}$ with the secondary antibody Alexa Fluor488-conjugated goat anti-rabbit lgG (1:500; Life Technologies, Monza, Italy, cat. N. A11008) in PBT (1:500; Life Technologies, Monza, Italy, cat. N. A11008). Cells were washed 3 times with PBT for 15 min each and counterstained with DAPI $(0.2 \mu \mathrm{g} / \mathrm{ml}$ in $1 \mathrm{X}$ PBS) for $5 \mathrm{~min}$. Slides were mounted in Vectashield (DBA 
Italia, Segrate, Italy, cat. N. H-1000). MIINSN, MII ${ }^{S N}$ and ovulated MII oocytes were immunostained as described above except for the primary antibody incubation which was performed for $1 \mathrm{hr}$ at $37^{\circ} \mathrm{C}$.

Oocytes of class I (10-30 $\mu \mathrm{m})$, class II (31-40 $\mu \mathrm{m})$ and class III (41-50 $\mu \mathrm{m})$ were transferred onto a slide and fixed in $10 \%$ formalin in 1X PBS at $4^{\circ} \mathrm{C}$ for $20 \mathrm{~min}$. The next steps were conducted as described above except for the following differences: all washes were done in PBT $(0.05 \%$ Tween-20 in 1X PBS); permeabilization was performed in $0.05 \%$ Triton $X-100$ in $1 X$ PBS for $20 \mathrm{~min}$, followed by $1 \mathrm{hr}$ incubation at $37^{\circ} \mathrm{C}$ in $0.1 \%$ BSA in PBT (0.05\% Tween-20 in 1X PBS); the secondary antibody was diluted 1:1000 in PBT (0.05\% Tween-20 in 1X PBS). For each class of oocytes, a number comprised between 10-60 oocytes, obtained from three independent experiments, was analysed.

\section{Image analysis}

Oocytes were examined with an Olympus Provis epifluorescence microscope equipped with single-bandpass filters for DAPI and Alexa488. Digital images were obtained with a cooled-coupled Photometrix $\mathrm{CH}-350$ controlled by the IPLab software used also to pseudo-color the images. Three-dimensional image (3D) analysis was performed on an Olympus BX60 equipped with a Tango motorized Z-axis (Märzhäuser Wetzlar, Wetzlar, Germany), epifluorescence and an Olympus DP72 digital camera. A collection of optical sections ( $0.5 \mu \mathrm{m} Z$-spacing) were captured and analysed using the Olympus Cellsens Dimension software.

\section{Acknowledgments}

We thank the following institutions for their support: Regione Lombardia (Fondo per la promozione di accordi istituzionali), Alma Mater Ticinensis (Promuovere la ricerca di eccellenza), Fondazione I.R.C.C.S. Policlinico San Matteo di Pavia, FIL 2008, FIL 2009.

\section{References}

CHOI M, LEE OH, JEON S, PARK M, LEE DR, KO JJ, YOON TK, RAJKOVIC A, CHOI $Y(2010)$. The oocyte-specific transcription factor, Nobox, regulates the expression of Pad6, a peptidylarginine deiminase in the oocyte. FEBS Lett 584: 3629-34.

CHOI Y, RAJKOVIC A (2006). Characterization of NOBOX DNA binding specificity and its regulation of Gdf9 and Pou5f1 promoters. J Biol Chem 281:35747-35756.
GENTILE L, MONTI M, SEBASTIANO V, MERICO V, NICOLAI R, CALVANI M, GARAGNA S, REDI CA, ZUCCOTTI M (2004). Single-cell quantitative RT-PCR analysis of Cpt1b and Cpt2 gene expression in mouse antral oocytes and in preimplantation embryos. Cytogenet Genome Res 105: 215-221.

INOUEA, NAKAJIMAR, NAGATAM, AOKIF (2008). Contribution of the oocyte nucleus and cytoplasm to the determination of meiotic and developmental competence in mice. Hum Reprod 23: 1377-1384.

LONGO F, GARAGNA S, MERICO V, ORLANDINI G, GATTI R, SCANDROGLIO R, REDI CA, ZUCCOTTI M (2003). Nuclear localization of NORs and centromeres in mouse oocytes during folliculogenesis. Mol Reprod Dev 66: 279-290.

RAJKOVICA, PANGASSA, BALLOWD, SUZUMORI N, MATZUK MM (2004). NOBOX deficiency disrupts early folliculogenesis and oocyte-specific gene expression. Science 305: 1157-1159.

THELIE A, PAPILLIER P, PERREAU C, UZBEKOVA S, HENNEQUET-ANTIER C, DALBIES-TRAN R (2009). Regulation of bovine oocyte-specific transcripts during in vitro oocyte maturation and after maternal-embryonic transition analyzed using a transcriptomic approach. Mol Reprod Dev 8: 773-82.

ZUCCOTTI M, BOIANI M, PONCE R, GUIZZARDI S, SCANDROGLIO R, GARAGNA $\mathrm{S}, \mathrm{REDI}$ CA (2002a). Mouse Xist expression begins at zygotic genome activation and is timed by a zygotic clock. Mol Reprod Dev 61: 14-20.

ZUCCOTTI M, GIORGI ROSSI P, MARTINEZ A, GARAGNA S, FORABOSCO A REDICA (1998). Meiotic and developmental competence of mouse antral oocytes. Biol Reprod 58: 700-704.

ZUCCOTTI M, MERICO V, SACCHI L, BELLONE M, BRINK TC, BELLAZZI R, STEFANELLI M, REDI CA, GARAGNA S, ADJAYE J (2008). Maternal Oct-4 is a potential key regulator of the developmental competence of mouse oocytes. BMC Dev Biol 8: 97.

ZUCCOTTI M, MERICO V, SACCHI L, BELLONE M, BRINK TC, STEFANELLI M, REDI CA, BELLAZZI R, ADJAYE J, GARAGNA S (2009a). Oct-4 regulates the expression of Stella and Foxj2 at the Nanog locus: implications for the developmental competence of mouse oocytes. Hum Reprod 24: 2225-2237.

ZUCCOTTI M, MERICO V, REDI CA, BELLAZZI R, ADJAYE J, GARAGNAS (2009b). Role of Oct-4 during acquisition of developmental competence in mouse oocyte. Reprod Biomed Online 19 Suppl 3: 57-62.

ZUCCOTTI M, PICCINELLI A, GIORGI ROSSI P, GARAGNA S, REDI CA (1995). Chromatin organisation during mouse oocyte growth. Mol Reprod Dev 41:479-485.

ZUCCOTTI M, PONCE RH, BOIANI M, GUIZZARDI S, GOVONI P, SCANDROGLIO R, GARAGNA S, REDI CA (2002b). The analysis of chromatin organisation allows selection of mouse antral oocytes competent for development to blastocyst. Zygote 10: 73-78. 


\section{Further Related Reading, published previously in the Int. J. Dev. Biol.}

Enhancing somatic nuclear reprogramming by Oct4 gain-of-function in cloned mouse embryos Martin J. Pfeiffer, Sebastian T. Balbach, Telma C. Esteves, Nicola Crosetto and Michele Boiani Int. J. Dev. Biol. (2010) 54: 1649-1657

Unaltered imprinting establishment of key imprinted genes in mouse oocytes after in vitro follicle culture under variable follicle-stimulating hormone exposure Ellen Anckaert, Tom Adriaenssens, Sergio Romero, Sarah Dremier and Johan Smitz Int. J. Dev. Biol. (2009) 53: 541-548

The 2-cell block occurring during development of outbred mouse embryos is rescued by cytoplasmic factors present in inbred metaphase II oocytes Mario Zanoni, Silvia Garagna, Carlo A. Redi and Maurizio Zuccotti Int. J. Dev. Biol. (2009) 53: 129-134

Developmental competence of immature pig oocytes under the influence of EGF, IGF-I, follicular fluid and gonadotropins during IVM-IVF processes M J Illera, P L Lorenzo, J C Illera and R M Petters Int. J. Dev. Biol. (1998) 42: 1169-1172

Location of DNA within the nucleolus of rat oocytes during the early stages of follicular growth

$M$ Thiry and G Goessens

Int. J. Dev. Biol. (1992) 36: 139-142

5 yr ISI Impact Factor $(2011)=2.959$

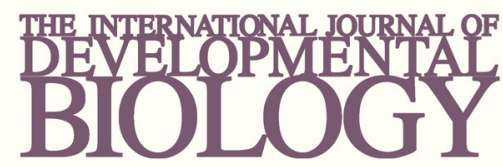

Volume 54 Nos. 6/7
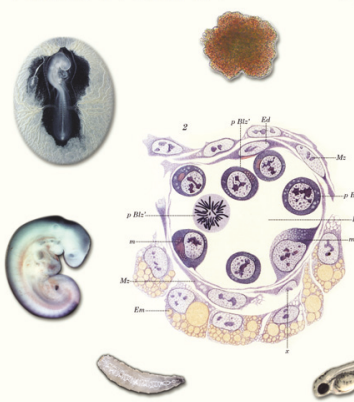

Developmental Hematopoiesis
Special Issue
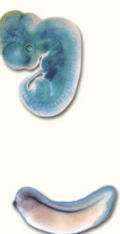

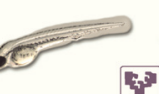

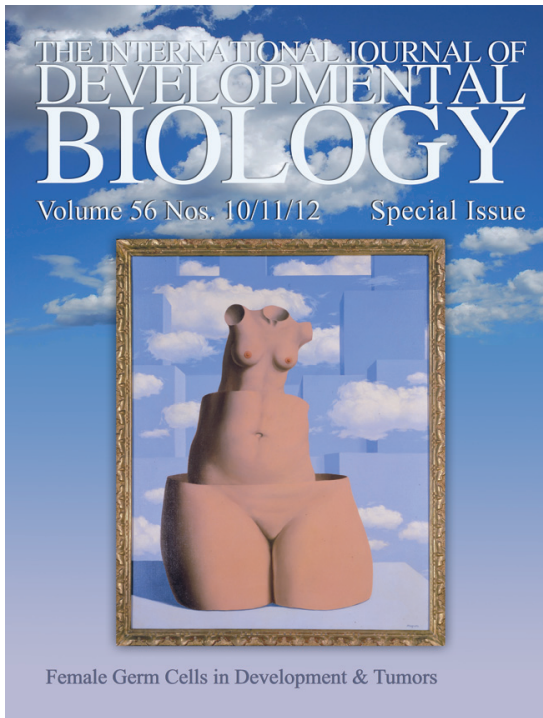
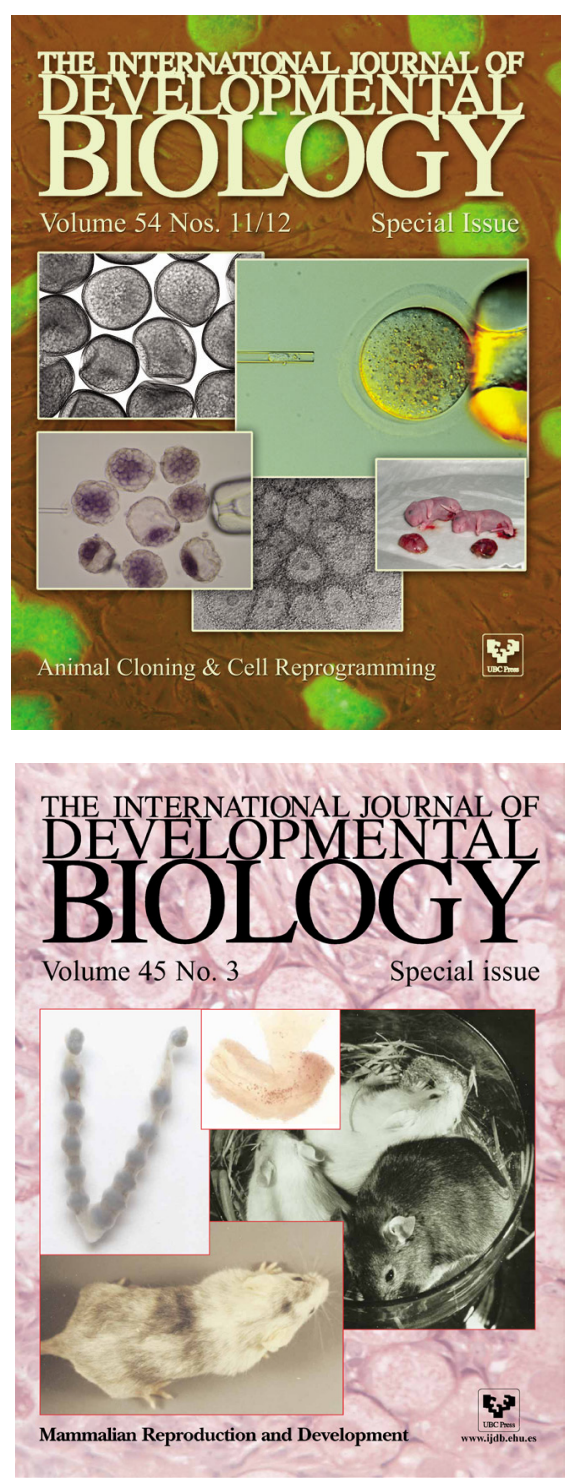